\title{
Current Trends in Application of Platelet Rich Fibrin
}

\section{Krishna Madhukumaran Pillai Sreekaladevi ${ }^{*}$, Shreya Kothari ${ }^{2}$, Srikanth Tatikonda ${ }^{3}$, Vaishnavi Ramanan ${ }^{3}$, Valliammai Rajendran ${ }^{4}$ and Hridya Jayaprakash ${ }^{5}$}

\author{
${ }^{1}$ MDS, Ex-Post Graduate, Department of Oral Medicine and Maxillofacial Radiology, \\ KVG Dental College and Hospital, Sulliya, India \\ ${ }^{2} B D S, M P H$, Emory University, Atlanta, Georgia, USA \\ ${ }^{3} B D S, M A S$, Vancouver, British Columbia, Canada \\ ${ }^{4} \mathrm{MDS}$, Periodontist, Trichy, Tamil Nadu, India \\ ${ }^{5}$ MDS, Periodontist, Clove Dental, Sholinganallur, Chennai, Tamil Nadu, India
}

*Corresponding Author: Krishna Madhukumaran Pillai Sreekaladevi, MDS, Ex-Post

Graduate, Department of Oral Medicine and Maxillofacial Radiology, KVG Dental

College and Hospital,Sulliya,India.
Received: November 22, 2021

Published: November 30, 2021

(C) All rights are reserved by Krishna

Madhukumaran Pillai Sreekaladevi., et al.

\begin{abstract}
Platelet rich fibrin (PRF) is an autologous biomaterial with growth factors and cytokines entrapped in a fibrin matrix. In recent times it has been used in various disciplines in dentistry in a wide range of treatment modalities. An electronic literature research using the biomedical search engine "National Library of Medicine" (PubMed-MEDLINE) was performed. This narrative review aims to summarize the relevant literature regarding the technique of using PRF, its preparation, advantages, and disadvantages of using it in clinical situations. PRF alone or in combination with other biomaterials seems to have several advantages and indications both for medicine and dentistry, as it is a minimally invasive technique with reduced risks and satisfactory clinical results.
\end{abstract}

Keywords: Platelet; Healing; Fibrin

\section{Introduction}

Reconstruction and regeneration of tissue has become the area of interest for researchers during the last few decades. Development of various biomaterials to improve the reconstruction procedures and wound healing has been well studied recently. Biomaterials such as bone grafts of different origin, alloplastic materials such as hydroxyapatite crystals have been experimented widely to evaluate their efficacy in regeneration and healing. However, all biomaterials have their own advantages and disadvantages $[1,2]$. This had led to the development of platelet rich fibrin (PRF) which is an autologous material shown to have excellent healing potential. This narrative review focuses on PRF and its current applications in dentistry in a wide view.

\section{Why platelets?}

Wound healing is a complex process which is promoted by cellular organization, chemical signals, and the extracellular matrix for tissue repair [3]. However, the mechanism of wound healing is not completely understood till date. Healing is initiated with clot formation, epithelialization, angiogenesis, and collagen formation. Growth factors play a crucial role in the above-mentioned processes. It is well known that platelets have the potential to promote healing as they contain growth factors. These growth factors are responsible for the following processes: 1 . Mitosis; 2 . Synthesis of collagen; 3 . Growth of blood vessels; 4 . Migration of cells to the site of injury; 5. Cell differentiation. Hence it is widely accepted that platelets are involved in tissue homeostasis and wound healing [4]. 
What is platelet rich fibrin (PRF)?

Platelet Rich Fibrin and Platelet Rich Plasma (PRP) are the commonly used platelet concentrates. PRF is a fibrin matrix comprising of cytokines, growth factors and cells which are entrapped in the matrix network and gets released slowly over time [5]. It acts as a resorbable membrane over the wound. The membrane releases growth factors for a period of 1 to 4 weeks which is considered as a crucial period for wound healing. PRF was first used by Choukroun in the year 2001 in the field of oral and maxillofacial surgery [6]. It is the second-generation platelet concentrate which is completely autologous and does not require any additives in it. This factor makes it different from PRP.

Preparation of PRF is a single standardized step as described by Choukroun. $10 \mathrm{ml}$ of venous blood is withdrawn from the patient and collected to a blood collection tube made of glass. Following this, the collected blood is centrifuged at $3000 \mathrm{rpm}$ for 10 minutes. Clot formation and fractioning are induced by centrifugal forces. The glass particles in the collection tube initiates clot formation and activate platelets [7]. At the end of centrifugation, 3 layers are formed in the test tube. The topmost layer is platelet poor plasma (PPP), the middle layer is the PRF and the bottom most layer contains red blood cells (Figure 1). The PRF layer is separated from the tube with the help of tweezers and separated from RBC layer to make it ready for use. The junction between the PRF layer and $\mathrm{RBC}$ layer has shown to have a lot of growth factors and hence it is also used (Figure 1). The procedure of centrifugation needs to be done as quickly as possible. Slow handling leads to blood coagulation and diffuse polymerization [8]. This affects the properties of the PRF membrane. Once the PRF is taken out of the tube, it appears like a jelly. The liquid content of it could be removed by compressing between two gauze pieces or using PRF kit to obtain even thickness of the membrane. The exudate from PRF also has various growth factors, hence can be used to hydrate biomaterials such as bone grafts [8].

\section{PRF in wound healing}

The fibrin mesh of PRF contains platelets, cytokines, leukocytes, and stem cells. It enhances tissue angiogenesis, epithelialization and granulation tissue maturation leading to excellent healing outcomes. Transforming Growth Factor beta (TGF-beta) and Platelet Derived Growth Factor (PDGF) are the two essential growth factors

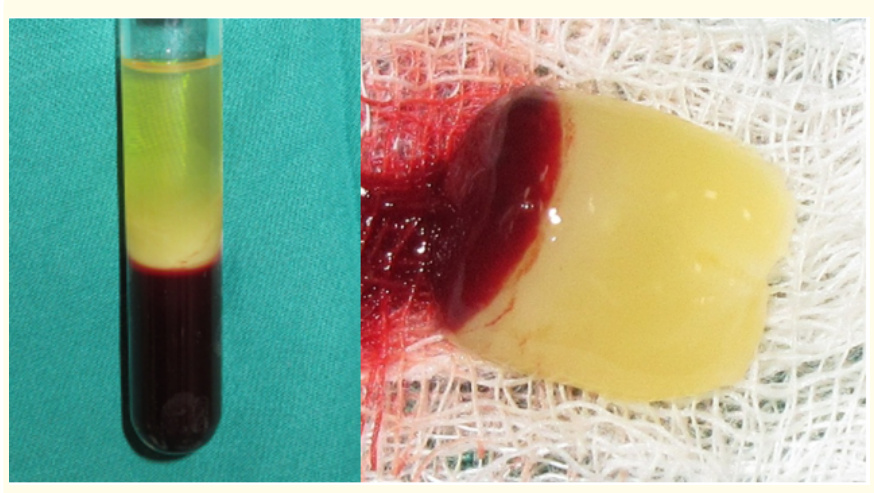

Figure 1: Platelet Rich Fibrin (PRF).

released by PRF [9]. PDGF promote osteoblastic cell proliferation and it is the first growth factor to reach the site of injury. TGF-beta is an inflammatory regulator, involved in fibroblast proliferation, eventually collagen formation. Vascular endothelial growth factor (VEGF) acts on endothelial cells and aids in angiogenesis [4]. The fibrin in PRF entraps surrounding stem cells into it and promotes neovascularization. The matrix guides the migration of epithelial and fibroblast cells to the wound site. Fibrin and fibronectin also help in collagen synthesis. PRF contains a variety of factors that promotes wound healing.

\section{Applications of PRF in dentistry}

Endodontics $[10,11]$

- $\quad$ PRF when used as a membrane could prevent the extrusion of the material.

- $\quad$ PRF scaffold in infected immature pulpal tissue can expect to cause pulpal regeneration and revitalization.

- $\quad$ PRF along with MTA in apexification procedure results in faster periapical wound healing, especially in large lesions.

- Used as a filling material in periapical bone defects.

- $\quad$ Also used in pulpotomy procedure along with GIC.

- $\quad$ PRF causes proliferation of pulpal cells and aids them to differentiate into odontoblast like cells.

- $\quad$ To regenerate pulp and dentin complex. 
Oral and maxillofacial surgery $[12,13]$

- As a filling material in extraction socket. It helps in blood clot stabilization and neovascularization. This can be used in immunocompromised patients where wound healing is compromised.

- As an adjunct to anticoagulant therapy.

- $\quad$ Can be used in sinus lift procedures with or without the addition of bone graft materials.

- $\quad$ Can also be combined with alloplastic materials such as tricalcium phosphate to elevate the sinus floor.

- Used in avulsion sockets especially when one or two bony wall is missing.

- The fibrin network in PRF holds the bone graft materials together in place. Hence displacement of graft material is avoided when PRF is used.

- In cases where approximation of tissue ends is not complete and in wide extraction sockets, PRF membrane can be used to cover the defect and prevent tissue necrosis.

- Used in guided bone regeneration (GBR) technique.

- $\quad$ Ridge augmentation technique.

- $\quad$ Socket preservation technique.

- $\quad$ Aids in healing in patients with Medication Related Osteonecrosis of the Jaw (MRONJ).

Periodontics $[13,14]$

- $\quad$ PRF along with bone graft materials is used in intrabony defects

- $\quad$ To treat endo-perio lesions.

- $\quad$ PRF membrane is used in guided tissue regeneration (GTR) technique.

- Can also be used in root coverage procedure as a sole material or in combination with graft materials.

- PRF releases growth factors for periodontal regeneration.
- $\quad$ Reduces post-operative pain as it promotes wound healing.

- $\quad$ Reduces probing depth and attachment loss when PRF is combined with open flap debridement.

\section{Orthodontics [15]}

- Use of PRF in orthodontic treatment remains limited. However, PRF membrane was tried in periodontally accelerated osteogenic orthodontics (PAOO) with and without bone grafts. PRF membrane helps in wound healing in the post-operative period following PAOO.

\section{Current levels of evidence of PRF}

PRF is a bioactive autologous system. Wide research in this field has demonstrated PRF as an effective drug delivery system [16]. The standardized protocol of PRF preparation uses a high-speed centrifugation. However, current research has shown that slow speed centrifugation makes PRF more effective when compared with high-speed centrifugation [17]. Higher number of leukocytes and platelets are found in PRF that is prepared with slow speed centrifugation. This makes PRF to have more immunologic potential and healing ability.

PRF when combined with other biomaterials for regeneration, it improves the efficacy of the added biomaterial. This is termed as 'biologization' of the biomaterial as it meets PRF prior to its application at the surgical site. PRF coated biomaterial promotes an increases cell to cell communication, adhesion and migration [18].

\section{Conclusion}

PRF is also effective in case of peri-implantitis. It helps in calcification of surrounding bone and in post-operative healing. A recent Cochrane review has concluded that PRF along with other biomaterials is effective in peri-implant tissue regeneration when compared with the sole use of the biomaterial [19]. Use of PRF during implant treatment reduces the post-operative pain and improves patient comfort. This brings positive attitude for patients towards dental treatment.

No significant differences were seen when PRF is used alone or in combination with other biomaterials for sinus floor elevation procedures. However, pocket depth reduction and attachment gain is significantly improved when PRF was combined with other biomaterials [20]. 


\section{Future Directions}

Though PRF is effective in healing and regeneration, more research is required to learn more about it. At present, only shortterm studies are available with PRF. Long term clinical control trials with more number of sample is required to pave way for the future. To promote reproducible studies with a higher scientific level of evidence, a standardization of PRF protocols is required to show the benefit of PRF in tissue regeneration. Along with the available clinical trials, histopathological studies are also required to understand about the nature of the regenerated tissue in the defect and to learn about the biology and efficacy of PRF more effectively.

\section{Declaration of Interest}

None.

\section{Acknowledgement}

None.

\section{Bibliography}

1. Duttenhoefer F., et al. "Follow-up of implant survival comparing ficoll and bone marrow aspirate concentrate methods for hard tissue regeneration with mesenchymal stem cells in humans". Bioresearch Open Access 3.2 (2014): 75-76.

2. Sakkas A., et al. "Autogenous bone grafts in oral implantology - is it still a "gold standard"? A consecutive review". International Journal of Implant Dentistry 3.1 (2017): 23.

3. Singer AJ and Clark RA. "Cutaneous wound healing". The New England Journal of Medicine 341.10 (1999): 738-746.

4. Kanakamedala A., et al. "Treatment of a furcation defect with a combination of platelet-rich fibrin (PRF) and bone graft - a case report". ENDO (London England) (2009): 127-135.

5. Gupta V., et al. "Regenerative Potential of Platelet Rich Fibrin in Dentistry: Literature Review". Asian Journal of Oral Health and Allied Sciences 14.6 (2011): 22-28.

6. Choukroun J., et al. "The PRF: an opportunity in implantology". Pantodontid (2001): 55-62.

7. Malathi K., et al. "Periodontal regeneration of an intrabody osseous defect with combination of platelet rich fibrin and bovine derived demineralized bone matrix: A case report". IOSRJDMS 4.2 (2013): 20-26.
8. Singh S., et al. "Application of PRF in surgical management of periapical lesions". National Journal of Maxillofacial Surgery 4.1 (2013): 94-99.

9. K. B. Jayalakshmi., et al. "Platelet-Rich Fibrin with $\beta$-Tricalcium Phosphate-A Novel Approach for Bone Augmentation in Chronic Periapical Lesion: A Case Report". Case Reports in Dentistry (2012).

10. Shivashankar VY., et al. "Platelet Rich Fibrin in the revitalization of tooth with necrotic pulp and open apex". Journal of Conservative Dentistry 15.4 (2012): 395-398.

11. I.B. Geeta., et al. "A Natural Meliorate: Revolutionary Tissue Engineering in Endodontics". Journal of Clinical and Diagnostic Research 7.11 (2013): 2644-2646.

12. Corso MD., et al. "Use of autologous leukocyte and platelet rich fibrin (L-PRF) membrane in post avulsion sites: an overview of Choukroun's PRF". The Journal of Implant and Advanced Clinical Dentistry (2010): 27-35.

13. Naik B., et al. "Role of Platelet rich fi brin in wound healing: A critical review". Journal of Conservative Dentistry 16.4 (2013): 284-293.

14. Qi Li., et al. "Platelet-Rich Fibrin Promotes Periodontal Regeneration and Enhances Alveolar Bone Augmentation". Biomedical Research International (2013).

15. Munoz F., et al. "Use of leukocyte and platelet-rich fibrin (LPRF) in periodontally accelerated osteogenic orthodontics (PAO0): clinical effects on edema and pain". Journal of Clinical and Experimental Dentistry 8.2 (2016): e119-e124.

16. Ghanaati S., et al. "Advanced platelet-rich fibrin: a new concept for cell-based tissue engineering by means of inflammatory cells". Journal of Oral Implantology 40.6 (2014): 679-689.

17. Choukroun J and Ghanaati S. "Reduction of relative centrifugation force within injectable platelet-rich-fibrin (PRF) concentrates advances patients' own inflammatory cells and platelets: first introduction of the low-speed centrifugation concept". European Journal of Trauma and Emergency Surgery 44.1 (2017): 87-95.

18. Al-maawi S., et al. "In vivo cellular reactions to different biomaterials-physiological and pathological aspects and their consequences". Seminars in Immunology Journal 29 (2017): 49-61. 
19. Esposito M., et al. "Treatment of periimplantitis: what interventions are effective? A Cochrane systematic review". European Journal of Oral Implantology 5 (2012): S21-S41.

20. Ghanaati S., et al. "Fifteen Years of Platelet Rich Fibrin in Dentistry and Oral maxillofacial Surgery: How High is the Level of Scientific Evidence?". Journal of Oral Implantology 44.6 (2018):

471-492.

Volume 5 Issue 12 December 2021

(C) All rights are reserved by Krishna Madhukumaran Pillai Sreekaladevi., et al. 